\title{
ON A NATURAL CONNECTION BETWEEN THE ENTROPY SPACES AND HARDY SPACE Re $H^{1}$
} ROMUALD DABROWSKI

(Communicated by J. Marshall Ash)

\begin{abstract}
In 1983 B. Korenblum $[7,8]$ introduced a class of Banach function spaces associated with the notion of entropy (we will call these spaces and their norms entropy spaces and entropy norms, respectively). Entropy spaces were used in [8] as a tool for proving a new convergence test for Fourier series which includes classical tests of Dirichlet-Jordan and Dini-Lipschitz.

In this paper we construct natural linear operators from the entropy spaces to Hardy space $\operatorname{Re} H^{1}[\mathbf{5}, 6]$. In fact, these operators define multiplier type bounded embeddings of entropy spaces to $\operatorname{Re} H^{1}$. As a corollary we obtain a growth condition for Fourier coefficients of a continuous periodic function of bounded entropy norm (as announced in [4]). In particular, we show that if $f$ is a continuous periodic function of bounded Shannon entropy norm (resp. of bounded Lipschitz entropy norm with exponent $q, 0<q<1$ ), and $\left\{a_{n}\right\}_{n \in \mathbf{Z}}$ are the Fourier coefficients of $f$, then $\sum_{n \neq 0}\left|a_{n}(\log (|n|) / n)\right|<\infty$ (resp. $\sum_{n \neq 0}\left|a_{n}\right| /|n|^{q}<\infty$ ). In $\S 4$ we study the relationship between the dual spaces of entropy spaces and space B.M.O. of functions of bounded mean oscillation. In $\S 5$ we conjecture that $\operatorname{Re} H^{1}$ is a direct limit of the entropy spaces in an appropriate sense.
\end{abstract}

1. Two representations of an entropy norm. Let $\mathbf{T}=\mathbf{R} / \mathbf{Z}$ be the unit circle, and let $|E|=\int_{E} d x$ denote the normalized Lebesgue measure of a Borel subset $E$ of $\mathbf{T}$. Also, let $\kappa(s), 0<s \leq 1$, be a positive nondecreasing concave function such that $\kappa(s)=1$. The $\kappa$-entropy of a finite subset $E$ of $\mathbf{T}(E \neq \varnothing)$ is $\kappa(E)=\sum_{i} \kappa\left(\left|I_{i}\right|\right)$, where $\left\{I_{i}\right\}$ are the complementary $\operatorname{arcs}$ of $E$ in $\mathbf{T}$. For an infinite subset $E$ of $\mathbf{T}$ we set $\kappa(E)=\sup \{\kappa(F) ; E \supset F, F$ finite $\}$. We also put $\kappa(\varnothing)=0$. The $\kappa$-entropy norm (first introduced in $[\mathbf{7}, 8]$ ) of a real continuous function $f$ on $\mathbf{T}$ is defined by formula $\|f\|_{\kappa}=\int_{\mathbf{R}} \kappa\left(f^{-1}(\{y\})\right) d y$.

THEOREM 1 [2]. Let $\kappa(s), 0<s \leq 1$, be a positive nondecreasing concave function such that $\kappa\left(0^{+}\right)=0$ and $\lim _{s \rightarrow 0}(\kappa(s) / s)=\infty$. There is a unique Borel probability measure $\mu_{\kappa}$ on the unit interval $(0,1]$ such that

$$
\|f\|_{\kappa}=\int_{\mathbf{T}} \int_{0}^{1} \frac{1}{s} \Omega_{I}(f) d x d \mu_{\kappa}(s),
$$

where $\Omega_{I}(f)$ is the oscillation of function $f$ on the arc $I=I(x, s)$ in $\mathbf{T}$ of length $s$ and center at $x$. A relationship between $\kappa$ and $\mu_{\kappa}$ is given by

$$
\kappa(s)=\int_{0}^{s} \int_{t}^{1} \frac{1}{u} d \mu_{\kappa}(u) d t .
$$

Received by the editors December 3, 1987.

1980 Mathematics Subject Classification (1985 Revision). Primary 42A20, 46E15. 
It is then proved in [2] that the set $\mathbf{C}_{\kappa}$ of real continuous functions on $\mathbf{T}$ of finite $\kappa$-entropy norm is a Banach algebra with respect to norm $\|\cdot\|_{\kappa}+\|\cdot\|_{\infty}$.

2. Natural embedding of space $\mathbf{C}_{\kappa}$ into Hardy space $\operatorname{Re} H^{1}$. We recall that a real function $a(t)$ on $\mathbf{T}$ is called an atom if (i) the support of $a(t)$ is contained in an subarc $I$ of $\mathbf{T}$; (ii) $|a(t)| \leq|I|^{-1}$ for all $t$ in $\mathbf{T}$; (iii) $\int_{\mathbf{T}} a(t) d t=0$. It is proved in [1] that

$$
\sup \left\{\|a\|_{\operatorname{Re} H^{1}}, a \text { atom }\right\}=1,
$$

for a suitable choice of norm of $\operatorname{Re} H^{1}$. From now on we fix a function $\kappa$ with properties assumed in Theorem 1 and we let $\mu=\mu_{\kappa}$ be the corresponding probability measure. Also, let $\chi_{I}$ denote the characteristic function of a subarc $I$ of $\mathbf{T}$. For any function $f, f \in \mathbf{C}_{\kappa}$, we put

$$
T_{\kappa} f(t)=\int_{\mathbf{T}} \int_{0}^{1} \frac{1}{s^{2}} \chi_{I}(t)(f(t)-f(I)) d \mu_{\kappa}(s) d x, \quad t \in \mathbf{T},
$$

where $I=I(x, s), x \in \mathbf{T}, 0<s \leq 1$, and $f(I)=(1 / s) \int_{I} f(x) d x$. The integral makes sense for almost all $t$ since

$$
\begin{aligned}
& \int_{\mathbf{T}} \int_{\mathbf{T}} \int_{0}^{1} \frac{1}{s^{2}} \chi_{I}(t)|f(t)-f(I)| d \mu_{\kappa}(s) d x d t \\
& \quad \leq \int_{\mathbf{T}} \int_{\mathbf{T}} \int_{0}^{1} \frac{1}{s^{2}} \chi_{I}(t) \Omega_{I}(f) d \mu_{\kappa}(s) d x d t=\|f\|_{\kappa}
\end{aligned}
$$

and Fubini's theorem applies. In fact, integral (2.2) defines a bounded linear operator from space $\mathbf{C}_{\kappa}$ to space $L^{1}(\mathbf{T})$ of all Lebesgue integrable functions on $\mathbf{T}$. In fact much more can be said about operator $\mathbf{T}_{\kappa}$. First observe that for any arc $I=I(x, s), x \in \mathbf{T}, 0<s \leq 1$, one can write

$$
\frac{1}{s} \chi_{I}(t)(f(t)-f(I))=\Omega_{I}(f) a_{I}(t) \quad t \in T,
$$

where $a_{I}(t)$ is an atom supported on $I$ and dependent on $f$. Hence integral (2.2) can be viewed as a Bochner integral [10] of a function

$$
(x, s) \rightarrow \frac{1}{s} \Omega_{I(x, s)}(f) a_{I(x, s)},
$$

defined on measure space $\left(\mathbf{T} \times(0,1], d x d \mu_{\kappa}\right)$ with values in $\operatorname{Re} H^{1}$. It is easy to see that this function is strongly continuous as a function on metric space $\mathbf{T} \times(0,1]$ with values in $L^{2}(\mathbf{T})$ and therefore, it is strongly continuous as a function with values in $\operatorname{Re} H^{1}$. Consequently, the function is weakly measurable and furthermore, since $\operatorname{Re} H^{1}$ is separable, it is also strongly measurable (see [10]). Now, the Minkowski's inequality for the norm of a Bochner integral (see [10]) and (2.1) yield the following result.

THEOREM 2. $T_{\kappa}$ is a bounded linear operator from space $\mathbf{C}_{\kappa}$ to Hardy space $\operatorname{Re} H^{1}$ such that $\left\|T_{\kappa} f\right\|_{\operatorname{Re} H^{1}} \leq\|f\|_{\kappa}$. 
3. Operator $T_{\kappa}$ as a multiplier. For any integer $n$ and $t$ in $\mathbf{T}$ we put $e_{n}(t)=$ $\exp (2 \pi$ int $)$. We fix $n, n \neq 0$, and we apply formula $(2.2)$ to function $e_{n}$ to obtain

$$
\begin{aligned}
T_{\kappa} e_{n}(t) & =\int_{0}^{1} \int_{0}^{1} \frac{1}{s^{2}} \chi_{I(x, s)}(t)\left(e_{n}(t)-e_{n}(I(x, s))\right) d \mu(s) d x \\
& =\int_{0}^{1} \frac{1}{s^{2}} d \mu(s)\left(\int_{t-s / 2}^{t+s / 2}\left(e_{n}(t)-\frac{e_{n}(x)}{2 \pi i n s}\left(e_{n}\left(\frac{s}{2}\right)-e_{n}\left(\frac{-s}{2}\right)\right)\right) d x\right) \\
& =\left(\frac{1}{2 \pi^{2} n^{2}} \int_{(0,1]}\left(\cos (2 \pi n s)-1+2 \pi^{2} n^{2} s^{2}\right) \frac{1}{s^{3}} d \mu(s)\right) e_{n}(t) .
\end{aligned}
$$

The latter integral is positive since the inequality $\cos (x)-1+x^{2} / 2>0$ holds for all $x, x \neq 0$. We have proved the following theorem.

THEOREM 3. Operator $T_{\kappa}$ is of multiplier type, i.e., $T_{\kappa} e_{n}=\beta_{n}(\kappa) e_{n}, n \in \mathbf{Z}$, where $\beta_{0}(\kappa)=0$ and

$$
\beta_{n}(\kappa)=\frac{1}{2 \pi^{2} n^{2}} \int_{(0,1]}\left(\cos (2 \pi n s)-1+2 \pi^{2} n^{2} s^{2}\right) \frac{1}{s^{3}} d \mu_{\kappa}(s), \quad n \neq 0 .
$$

Moreover, $\beta_{n}(\kappa)=\beta_{-n}(\kappa)>0$ for $n>0$ and $T_{\kappa}$, restricted to $\mathbf{C}_{\kappa}(0)=\{f \in$ $\left.\mathbf{C}_{\kappa} ; \int_{\mathbf{T}} f(t) d t=0\right\}$, is injective.

It is a well-known result of Hardy [5] that if $\sum_{n} c_{n} e_{n}$ is the Fourier series of a function $h \in \operatorname{Re} H^{1}$ then $\sum_{n \neq 0}\left|c_{n} / n\right|<C\|h\|_{\operatorname{Re} H^{1}}$ for certain constant $C>0$, independent on $h$. This fact together with Theorems 1 and 2 implies the following.

COROLLARY. Let $f$ be a continuous function of bounded $\kappa$-entropy, and if $\sum_{n} a_{n} e_{n}(t)$ is the Fourier series of $f$, then $\sum_{n \neq 0} \beta_{n}(\kappa)\left|a_{n} / n\right| \leq C\|f\|_{\kappa}$.

EXAMPLES.

1. If $\kappa(s)=s$, then $\mu$ is the point mass measure $\delta_{1}$ concentrated at $s=1$ and $\beta_{n}(\kappa)=1$ for any $n \neq 0$. Note that in this case $\|f\|_{\kappa}$ is just the oscillation of a function $f$ on $\mathbf{T}$.

2. Suppose that $d \mu(s)=q s q^{-1} d s$, for some $q, 0<q \leq 1$. Then a repeated integration by parts leads to the following formula:

$$
\beta_{n}(\kappa)=\frac{q}{(q-2)(q-3)}\left(q-4+2 n^{1-q} \int_{0}^{n}(1-\cos (2 \pi y)) y^{q-2} d y\right), \quad n>0 .
$$

In particular, if $\kappa(s)=s(|\log (s)|+1)$ is the Shannon entropy, then $d \mu(s)=d s$ (see [2]) and (3.2) give

$$
\beta_{n}(\kappa)=\int_{0}^{n}(1-\cos (2 \pi y)) y^{-1} d y-\frac{3}{2}, \quad n \neq 0 .
$$

Now, it is not difficult to prove that in this case $\lim _{n \rightarrow \infty}\left(\beta_{n}(\kappa) / \log (n)\right)=1$. Therefore, using Theorem 3, we conclude that if $\sum_{n} a_{n} e_{n}$ is the Fourier series of a continuous function of bounded Shannon entropy norm, then $\sum_{n \neq 0} \mid a_{n}(\log (|n|) / n \mid<\infty$. Similarly, formula (3.2) implies that if $\left\{a_{n}\right\}_{n \in \mathbf{Z}}$ are the Fourier coefficients of a continuous function with bounded Lipschitz entropy norm (i.e., $\kappa(s)=s^{q}, 0<q<1$, and by [2] $\left.d \mu(s)=q d \delta_{1}(s)+q(q-1) s^{q-1} d s\right)$, then

$$
\sum_{n \neq 0}\left|a_{n}\right| /|n|^{q}<\infty
$$


4. Natural operators from the space B.M.O to the dual spaces of the entropy spaces. Again, as in $\S 2$, we fix a function $\kappa$ and the corresponding probability measure $\mu=\mu_{\kappa}$. The probability representation of entropy norms given in [2] leads to the following description of the dual space $\left(\mathbf{C}_{\kappa}\right)^{*}$ of entropy space $\mathbf{C}_{\kappa}$.

THEOREM 4 [3, PROPOSITION 3.1]. Let $F$ be a continuous linear functional on $\mathbf{C}_{\kappa}$. There exists a set of signed measures $\eta=\left\{\eta_{I} ; I\right.$ a subarc of $\left.\mathbf{T}\right\}$ on $\mathbf{T}$, such that

(i) Each measure $\eta_{I}$ is concentrated on interval $I$ and $\int_{I} d \eta_{I}(x)=0$ and $\sup \left\{\operatorname{Var}\left(\eta_{I}\right) ; I\right.$ a subarc of $\left.\mathbf{T}\right\}<\infty$ (here Var stands for the total variation).

(ii) For every function $f$ in $\mathbf{C}_{\kappa}$ the map

$$
(x, s) \rightarrow \frac{1}{s} \int_{I(x, s)} f(y) d \eta_{I(x, s)}(y)
$$

is a real-valued integrable function on the measure space $\left(\mathbf{T} \times(0,1], d x d \mu_{\kappa}\right)$.

(iii)

$$
\langle F, f\rangle=\int_{\mathbf{T}} \int_{0}^{1} \frac{1}{s} \int_{I(x, s)} f(y) d \eta_{I(x, s)}(y) d \mu(s) d x .
$$

(iv) $\|F\|_{\left(\mathbf{C}_{\kappa}\right)^{*}}=\sup \left\{\operatorname{Var}\left(\eta_{I}\right) ;\right.$ I a subarc of $\left.\mathbf{T}\right\}$.

Conversely, any collection of measures $\eta=\left\{\eta_{I} ; I\right.$ a subarc of $\left.T\right\}$ satisfying conditions (i) and (ii) defines an element of space $\left(\mathbf{C}_{\kappa}\right)^{*}$ via formula (iii).

We recall that a real function $g(x), x \in T$, is of bounded mean oscillation (shortly, $g \in$ B.M.O) if $g$ is integrable and

$$
\|g\|_{\text {B.M.O }}=\sup \left\{\frac{1}{|I|} \int_{I}|g(x)-g(I)| d x ; I \text { a subarc of } T\right\}<\infty .
$$

It is clear that the set of measures $\eta(g)=\left\{\eta_{I}^{g}\right\}$, where

$$
d \eta_{I}^{g}(t)=\frac{1}{|I|} \chi_{I}(t)(g(t)-g(I)) d t
$$

satisfies conditions (i) of Theorem 4. Condition (ii) is also satisfied since the map $(x, s) \rightarrow \int_{I(x, s)} f(t) d \eta_{I(x, s)}^{g}(t)$ can be easily shown to be continuous. Consequently, the formula

$$
\left\langle S_{\kappa}(g), f\right\rangle=\int_{\mathbf{T}} \int_{0}^{1} \frac{1}{s} \int_{I(x, s)} f(t) d \eta_{I(x, s)}^{g}(t) d t d \mu_{\kappa}(s) d x, \quad g \in \text { B.M.O, } f \in \mathbf{C}_{\kappa},
$$

defines a bounded linear operator $S_{\kappa}$ from B.M.O to the dual of space $\mathbf{C}_{\kappa}$ with the operator norm at most 1 .

PROPOSITION. Let $f(t)$ and $g(t), t \in \mathbf{T}$, be a function in $\operatorname{Re} H^{1}$ and a function of bounded mean oscillation, respectively.

(i) If the product of $g$ and $T_{\kappa} f$ is in $L^{1}(\mathbf{T})$, then

$$
\int_{\mathbf{T}} g(t)\left(T_{\kappa} f\right)(t) d t=\left\langle S_{\kappa}(g), f\right\rangle \text {. }
$$

(ii) If $f_{1} \in \mathbf{C}_{\kappa_{1}}$ and $f_{2} \in \mathbf{C}_{\kappa_{2}}$ satisfy $T_{\kappa_{1}} f_{1}=T_{\kappa_{2}} f_{2}$ in $\operatorname{Re} H^{1}$ then $\left\langle S_{\kappa_{1}}(g), f_{1}\right\rangle=$ $\left\langle S_{\kappa_{2}}(g), f_{2}\right\rangle$ for any function $g$ of bounded mean oscillation.

We will need the following lemma in the proof of the proposition. 
LEMMA. Let $g$ and $I$ be an integrable function on $\mathbf{T}$ and a subarc of $\mathbf{T}$, respectively. For any $N, N \leq \infty$, we define

$$
N_{g(t)}= \begin{cases}N & \text { if } g(t)>N \\ g(t) & \text { if } g(t) \leq N\end{cases}
$$

and if $N \geq 0$ then we define

$$
g_{N}(t)= \begin{cases}N & \text { if } g(t)>N \\ -N & \text { if } g(t)<-N \\ g(t) & \text { if }|g(t)| \leq N\end{cases}
$$

Then

(i) $\int_{I}\left|{ }^{N} g(t)-{ }^{N} g(I)\right| d t \leq \int_{I}|g(t)-g(I)| d t$ for any level $N$.

(ii) If $g \in$ B.M.O then

$$
\left\|g_{N}\right\|_{\text {B.M.O }} \leq\left\|^{N} g\right\|_{\text {B.M.O }} \leq\|g\|_{\text {B.M.O }}
$$

for any positive level $N$.

PROOF OF THE LEMMA. It follows directly from the definition of the bounded mean oscillation norm and identity $g_{N}={ }^{N}\left(-{ }^{N} g\right)$ that (i) implies (ii). The identities $\left({ }^{N} g\right)+c={ }^{N+c}(g+c)$, and $\alpha\left({ }^{N} g\right)={ }^{\alpha N}(\alpha g)$ where $N, c$ and positive $\alpha$ are fixed constants, reduce the proof of (i) to the case when $g(I)=0$ and $|I|=1$. In this case, $\int_{I}|g(t)-g(I)| d t=2 \int_{I}-\left({ }^{0} g(t)\right) d t$, and ${ }^{N} g(I) \leq 0$. On the other hand, for any numbers $M$ and $N, M<N \leq \infty$, the following formulae hold:

$$
\begin{gathered}
\left|{ }^{N} g-M\right|={ }^{N} g-{ }^{M} g+M-{ }^{M} g={ }^{N} g+M-2\left({ }^{M} g\right), \\
{ }^{N} g(t)-N \leq{ }^{M} g(t)-M \text { for all } t \in T .
\end{gathered}
$$

Applying (4.2) in the case of $M={ }^{N} g(I)=\int_{I}^{N} g(t) d t$ and (4.3) in the case of $N=0$ we obtain the desired inequality

$$
\left.\int_{I}\right|^{N} g(t)-{ }^{N} g(I)\left|d t=2 \int_{I}\left(M-{ }^{M} g(t)\right) d t \leq 2 \int_{I}-\left({ }^{0} g(t)\right) d t=\int_{I}\right| g(t)-g(I) \mid d t .
$$

REMARK. Essentially the same proof shows that part (i) of the lemma remains true in the general case of a real function $g$ integrable over a probability space. The statement of the lemma appeared first in [9].

ProOF OF Proposition. Let $f$ and $g$ be a function in $\operatorname{Re} H^{1}$ and a bounded function, respectively. Then

$$
\begin{aligned}
\int_{\mathbf{T}} g(t) & \left(T_{\kappa} f\right)(t) d t \\
= & \int_{\mathbf{T}} \int_{0}^{1}\left(\int_{I(x, s)} \frac{1}{s^{2}}(g(t)-g(I(x, s)))(f(t)-f(I(x, s))) d t\right) d \mu(s) d x \\
= & \int_{\mathbf{T}} \int_{0}^{1}\left(\int_{I(x, s)} \frac{1}{s^{2}}(g(t)-g(I(x, s))) f(t) d t\right) d \mu(s) d x=\langle S \kappa(g), f\rangle
\end{aligned}
$$

since

$$
\int_{I} g(t)(f(t)-f(I)) d t=\int_{I}(g(t)-g(I)) f(t) d t \quad \text { for any subarc } I
$$


The change of the order of integration is justified, since $g$ is bounded and $f$ is of bounded $\kappa$-entropy norm (see the argument in the beginning of $\S 2$, showing that $T_{\kappa} f$ is an integrable function). Hence the identity (4.1) holds in the case of $g$ bounded and $f$ any function of bounded $\kappa$-entropy norm. Assume now that the product of $g$ and $T_{\kappa} f$ is integrable. Then

$$
\int_{\mathbf{T}} g(t)\left(T_{\kappa} f\right)(t) d t=\lim _{N \rightarrow \infty} \int_{\mathbf{T}} g_{N}(t)\left(T_{\kappa} f\right)(t) d t
$$

by the Lebesgue's dominated convergence theorem. On the other hand one has

$$
\begin{aligned}
\lim _{N \rightarrow \infty} & \left\langle S_{\kappa}\left(g_{N}\right), f\right\rangle \\
& =\lim _{N \rightarrow \infty} \int_{\mathbf{T}} \int_{0}^{1}\left(\int_{I(x, s)} \frac{1}{s^{2}}\left(g_{N}(t)-g_{N}(I(x, s))\right) f(t) d t\right) d \mu(s) d x \\
& =\lim _{N \rightarrow \infty} \int_{\mathbf{T}} \int_{0}^{1}\left(\int_{I(x, s)} \frac{1}{s^{2}}\left(g_{N}(t)-g_{N}(I(x, s))\right)(f(t)-f(I(x, s))) d t\right) d \mu(s) d x \\
& =\int_{\mathbf{T}} \int_{0}^{1}\left(\int_{I(x, s)} \frac{1}{s^{2}}(g(t)-g(I(x, s)))(f(t)-f(I(x, s))) d t\right) d \mu(s) d x \\
& =\left\langle S_{\kappa}(g), f\right\rangle
\end{aligned}
$$

for any $g$ in B.M.O and $f$ of bounded $\kappa$-entropy norm. The passage with the limit under the integral sign is justified since the lemma implies

$$
\begin{aligned}
\int_{I(x, s)} & \left.\frac{1}{s^{2}}\left|\left(g_{N}(t)-g_{N}(I(x, s))\right)(f(t)-f(I(x, s)))\right| d t\right) d \mu(s) d x \\
\leq & \frac{1}{s}\|g\|_{\text {B.M.O. }} \Omega_{I(x, s)}(f)
\end{aligned}
$$

and the Lebesgue dominated convergence theorem applies. The proof of the part (i) of the proposition is now complete since we have already shown that $\left\langle S_{\kappa}\left(g_{N}\right), f\right\rangle=$ $\int_{\mathbf{T}} g_{N}(t)\left(T_{\kappa} f\right)(t) d t$ for any $N \geq 0, g \in$ B.M.O and $f \in \mathbf{C}_{\kappa}$.

Suppose now that $f_{1} \in \mathbf{C}_{\kappa_{1}}$ and $f_{2} \in \mathbf{C}_{\kappa_{2}}$ satisfy $h=T_{\kappa_{1}} f_{1}=T_{\kappa_{2}} f_{2}$ in $\operatorname{Re} H^{1}$. Then the part (ii) of the proposition holds since one has

$$
\begin{aligned}
\left\langle S_{\kappa_{1}}(g), f_{1}\right\rangle & =\lim _{N \rightarrow \infty}\left\langle S_{\kappa_{1}}\left(g_{N}\right), f_{1}\right\rangle=\lim _{N \rightarrow \infty} \int_{\mathbf{T}} g_{N}(t) h(t) d t \\
& =\lim _{N \rightarrow \infty}\left\langle S_{\kappa_{2}}\left(g_{N}\right), f_{2}\right\rangle=\left\langle S_{\kappa_{2}}(g), f_{2}\right\rangle .
\end{aligned}
$$

\section{Questions.}

QUESTION 1. Is it true that if $h \in \operatorname{Re} H^{1}$ then there exists $\kappa$ and $f \in \mathbf{C}_{\kappa}$ such that $h=T_{\kappa} f$ and $\|h\|_{\operatorname{Re} H^{1}}=\|f\|_{\kappa}$ ? This would give an "integral" atomic decomposition of $h$ as defined by the formula (2.2). Also, if combined with results of $\S 4$, it would imply the Fefferman's duality

$$
\left(\operatorname{Re} H^{1}(0)\right)^{*}=\text { B.M.O. }
$$

QUESTION 2. We will write that for any two entropy functions $\kappa_{1}$ and $\kappa_{2}$, $\kappa_{1} \leq \kappa_{2}$ if there exists a bounded operator $T_{\kappa_{1} \kappa_{2}}: \mathbf{C}_{\kappa_{1}} \rightarrow \mathbf{C}_{\kappa_{2}}$ such that $T_{\kappa_{1}}=$ $T_{\kappa_{2}} \circ T_{\kappa_{1} \kappa_{2}}$. Give an explicit condition on $\kappa_{1}$ and $\kappa_{2}$ so that $\kappa_{1} \leq \kappa_{2}$. Is the relation 
" $\leq$ " a partial ordering in the set of entropy functions? If yes, is $\operatorname{Re} H^{1}(0)$ the direct limit of the system

$$
\left\{\mathbf{C}_{\kappa}, T_{\kappa_{1} \kappa_{2}}: \mathbf{C}_{\kappa_{1}} \rightarrow \mathbf{C}_{\kappa_{2}} \text { if } \kappa_{1} \leq \kappa_{2}\right\} ?
$$

\section{REFERENCES}

1. R. R. Coifman, A real variable characterization of $H^{p}$, Studia Math. 51 (1974), 269-274.

2. R. Dabrowski, Probability measure representation of norms associated with the notion of entropy, Proc. Amer. Math. Soc. 90 (1984), 263-268.

3. __ Bochner integral and continuous functions of bounded entropy, Preprint, 1986.

4. - On Fourier coefficients of a continuous periodic function of bounded entropy norm, Bull. Amer. Math. Soc. (N.S.) 18 (1988), 49-51.

5. P. Duren, Theory of $H^{p}$ spaces, Academic Press, New York, 1970.

6. P. Koosis, Introduction to $H^{p}$ spaces, London Math. Soc. Lecture Notes Series, no. 40, Cambridge Univ. Press, 1980.

7. B. Korenblum, On a class of Banach spaces associated with the notion of entropy, Trans. Amer. Math. Soc. 290 (1985), 527-553.

8. B. Korenblum, A generalization of two classical convergence tests for Fourier series and some new Banach spaces of functions, Bull. Amer. Math. Soc. (N.S.) 9 (1983), 215-218.

9. C. Sunberg, Truncations of B.M.O. functions, Indiana Univ. Math. J. 33 (1984).

10. K. Yosida, Functional analysis, Springer-Verlag, 1974, pp. 130-136.

Department of Mathematics, Columbia University, New York, New York 10027

Current address: Department of Mathematics, Indiana University, Bloomington, Indiana 47405 